\title{
Pelvic reconstruction with pedicle screw-rod system following pelvic resection type I/II/III in pelvic
} sarcoma

\begin{abstract}
1.1 Background: Reconstruction of pelvic after malignant tumor resection remains a major challenge in orthopedic oncology surgery. To restore weight bearing along anatomical axis while preserving motions is the aim after reconstruction to improve functional outcome thus provide quality of life. The pedicle screw-rod system has proven to be one of acceptable method of reconstructive surgery after pelvic type I, I+IV, and type IV resection due to less risk of complication and satisfactory functional outcome. However, none has been reported the outcome following other type of pelvic resection.
\end{abstract}

1.2 Methods: We performed hinged arthrodesis using the pedicle screw-rod system (PSRS) to reconstruct the pelvis following pelvic resection type I/II/III in a case of grade 2 Chondrosarcoma of right pelvic involving ilium and extending to the pubic rami. We elaborated techniques, functional outcome and complication following the procedure in 1 year follow up.

1.3 Results: The musculoskeletal tumor society score was 16 and 21 in 6 months and 1 year follow up respectively. Hip motion was $0-45$ degrees flexion post operatively. No complications such as infections, deep vein thrombosis, implant loosening and tumor recurrence, were seen in 1 year follow up.

1.4 Conclusions: We recommend the use of pedicle screw-rod system after pelvic resection type 1/II/III. However, longer period of follow up and larger number of cases treated are needed.

Keywords: pedicle screw-rod system, pelvic resection, hemipelvectomy, pelvic tumor, reconstruction
Volume 9 Issue 4 - 2019

\author{
Yogi Prabowo, M Alvin Shiddiqie Pohan \\ Department of Orthopaedic and Traumatology, Cipto \\ Mangunkusumo Hospital, Indonesia
}

Correspondence: MAlvin Shiddiqie Pohan, Department of Orthopaedic \& Traumatology, Cipto Mangunkusumo National Central Hospital, Indonesia, Tel +6287888744343, Email lvnpohan@gmail.com

Received: August 13,2019 | Published: August 29, 2019

\section{Introduction}

Malignant tumors involving pelvic region poses major problem for orthopedic oncologists since these tumors are usually largesized, extend to pelvic visceral organs and they tend to present late. ${ }^{1,2}$ Previously, the main stay treatment of malignant pelvic tumors was by limb ablation surgery or previously known as external hemipelvectomy. Currently with advancement of surgical techniques, chemotherapy and radiation therapy, limb salvage procedures by pelvic resection (internal hemipelvectomy) become the treatment of choice particularly if the tumor has not invaded major neuro vascular structures. However, the significant resultant defects after resection increases risk of mechanical failure due to collapse of residual bone segment during weight bearing. Achieving an adequate surgical margin is necessary for improving the oncological outcome of pelvic sarcomas but restoring anatomic alarch after the wide resection while retaining function is time consuming, technically demanding and complications are common. Thus, careful selection of the reconstruction method is needed. ${ }^{3,4}$

Pelvic reconstruction after malignant tumor resection is challenging due to the complexity of pelvic anatomy which demands an extensive exposure to preserve major neurovascular bundle. ${ }^{5}$ There are various reconstruction options have been reported such as bonegrafts (massive or in composite form with prosthesis), fixation with plates and screws composites (bone cement) and any kind of hip prosthesis (custom-made endoprosthesis combined with hip arthroplasty, saddle prosthesis or a modular hemipelvic prosthesis). However, these different methods have a high complication rate associated with dislocation, loosening, infection which leads to implant failure. ${ }^{6}$

Pedicle-screw and rod system (PSRS) with various techniques have been proposed and thought to have benefit in allowing some residual motion while still providing a solid construction of pelvic girdle. However, reports of outcome include functional outcome and evidence of complication following this method are still lacking. Lin et al has been reported about good mechanical outcomes of patient underwent pelvic reconstruction following pelvic resection type I, I+IV, and type IV. None has been reported about the outcome following other type of pelvic resection which reconstructed by pedicle screw-rod system. ${ }^{5,6}$

In this report, we present a case of grade 2 chondrosarcoma of right pelvic involving ilium, ischium and pubic rami treated by pelvic resection type I/II/III and reconstructed by PSRS. We evaluated the functional outcome and complication rate following treatment. The aim of this report is to elaborate relatively less complicated surgical techniques which applicable in many centers to reduce the intraoperative morbidity due to prolonged surgery duration. 


\section{Material and methods}

We present a 20 -year-old female patient with low grade spindle cell neoplasm of right pelvic involving ilium, ischium and pubic rami established by clinical, radiological, and histopathological finding in the clinicopathological conference (CPC). Based on CPC, in accordance with the classification systems of Enneking and Dunham, the type of resection is pelvic resection type I/II/III and reconstructed with hinged arthrodesis by pedicle screw-rod systems. Margin of resection was re-confirmed by histopathological analysis. Functional outcome according to musculoskeletal tumor society (MSTS) score and any complications were recorded during 6 months and one year post-operative period.

Before the surgery, patient had bowel preparation. Pre-operative antibiotics were administered. Patient was placed in lateral position on the contralateral side under general anaesthesia to allow backward and forward movement of the patient during the procedure. The utilitarian incision was carried over the iliac crest, extended to posterior superior iliac spine (PSIS), anterior superior iliac spine
(ASIS), and caudally to the subtrochanter femur. Anterior incision exposed external iliac artery, vein and femoral nerve which then preserved laterally. Abdominal muscles were detached. Origin of adductor magnus and pectineus muscle was transected and reflected distally while urethra was protected by retractor. Tensor fascia lata and muscle groups inserting to ASIS and anteroinferior iliac spine were dissected to expose the subtrochanter femur. Dissection was deepened subperiosteally to the major sciatic notch and to sacro-iliac joint posteriorly. The tumor mass was freed from surrounding tumorfree tissue and pelvic visceral organs. Osteotomy was performed through the symphysis pubic, supra-acetabular iliac wing, and subtrochanter femur.

The reconstruction began with monoaxial pedicle screw fixation to the remaining ilium, contralateral symphysis pubis and polyaxial pedicle screw for subtrocanterfemur. Two pre-bendrods were then applied to bridge the fixation and were augmented with antibioticimpregnated bone cement. After the final construct of pelvic bone, reconstruction of hip abductors was done to add more stability (Figure $1)$.
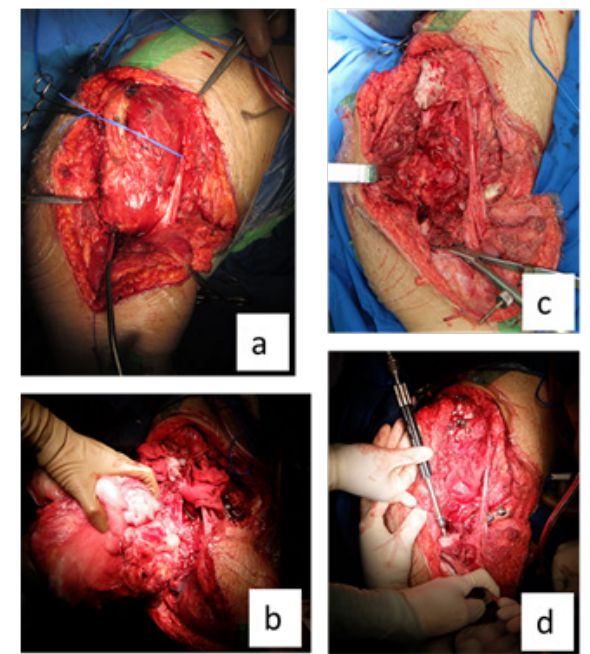

Figure I Pelvic Resection type II-III and reconstruction. a. tumor exposed; b. tumor removal; c. defect after resection; d. application of pedicle-screw and rod system; e. final construct.

\section{Results}

The surgery lasted for 12 hours with $4000 \mathrm{ml}$ blood loss. We performed wide marginal excision Post-operative $\mathrm{X}$ ray showed

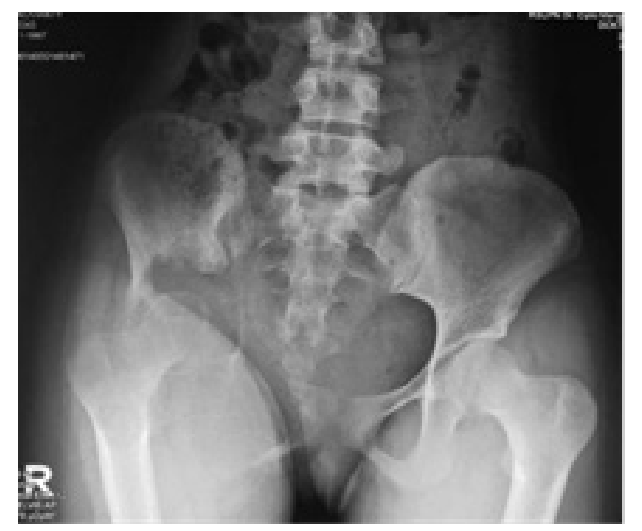

interconnecting pedicle and rodsystem reconstructed the pelvicarch (Figure 2). The histopathological examination revealed a grade 2 chondrosarcoma with superior, medial, lateral and distal margin were tumor-free.

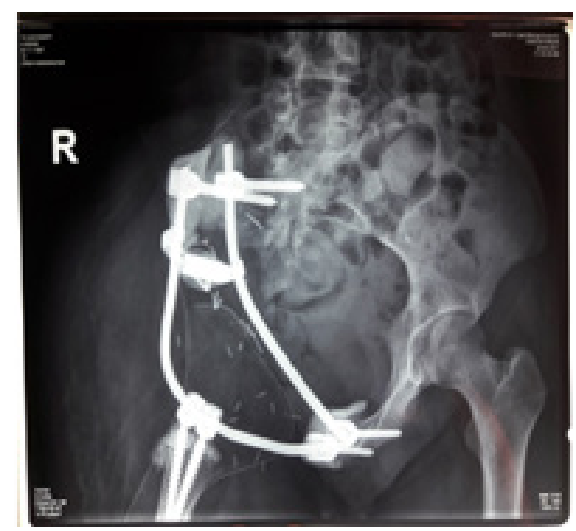

Figure 2 Pelvic AP plain radiograph. a. Pre-operative; b. Post-operative 
Post-operative range of motion was $0-45$ degrees hip flexion and patient could walk with crutches. Limb length discrepancy was $1 \mathrm{~cm}$. There was drop foot suggesting sciatic nerve palsy post-operatively. Patient was treated by medical rehabilitation department for regaining muscle strength and mobilization using ankle-foot orthosis. No other complications such as dislocation, deepvein thrombosis, emboli, infection, implant failure, local recurrence or distant metastases were observed in 6 months and 1 year follow up. The MSTS score was 16 and 21 in 6 months and 1 year follow up suggesting an improving functional outcome (Table 1).

Table I Patient functional score in 6-months and I-year post-operative period according to MSTS score

\begin{tabular}{lll}
\hline \multirow{2}{*}{ Parameters } & \multicolumn{2}{l}{ Post-operative period } \\
\cline { 2 - 3 } & 6 months & I year \\
\hline Pain & 4 & 5 \\
Function & 2 & 3 \\
Emotional & 3 & 5 \\
Support & 0 & 1 \\
Walking & 4 & 4 \\
Gait & 3 & 3 \\
Total & 16 & 21 \\
\hline
\end{tabular}

\section{Discussion}

Traditionally, limb ablation was the main stay treatment to treat pelvic malignant tumor. The rationale was to reduce local recurrence. Currently, Shin et al. Study showed that limb-salvage resection and hemipelvectomy were associated with an equal risk of contaminated margins. Thus, hind quarter amputation does not guaranteee radication of local disease. Joel et al. Also concluded that although the mortality is relatively low in hemipelvectomy group, the morbidity is high including wound infection and flapnecrosis. ${ }^{1,5-9}$

Despite of difficulty in obtaining negative margin of resection, limb salvage surgery for pelvic tumors can improve patient's quality of life by reducing psychological trauma and physical disability. Limb salvage surgery by pelvic resections are challenging procedure to manage pelvic malignancies, severe trauma, and infection. The primary goal is to preserve the ipsilateral lower extremity. This procedure includes the resection of the involved hemipelvis which further divided into four types according to Enneking and Dunham: type I involves the ilium; type II involves the acetabulum, type III involves the ischium and pubic rami, and type IV involves sacrum. ${ }^{1,5-7,10,11}$

In our patient, the tumor was extended from ilium, ischium to the ipsilateral pubic bones owe decided to perform wide excision involving those bone by pelvic resection type I/II/III.

Pelvic complex structure makes the pelvic resection is such a challenging and technically demanding surgery. Surgeon must identify and preserve pelvic viscera and neurovascular bundles. Massive blood lost urges patient to have massive transfusion and intensive care post operatively. However, preservation of the limb without retaining reasonable function is unsatisfactory. To reconstruct pelvic arch so that can over come weight while preserving some motion to gain better functional outcome must be considered which requires an adequate pre-operative planning. ${ }^{1,5,6,10,12}$
Adequate patient-based consideration of surgical options must be made considering the lowest risk of complication and allowed most rapid rehabilitation. Biological reconstruction is commonly used although there are some risks include non union, bone graft fracture and infection. Bone grafts either vascularized or non-vascularized are commonly used. However, these techniques require more complex procedures, longer surgery time, greater surgical trauma and greater risk of donor site morbidity. Alternatively, the functional reconstruction with the PSRS now emerging as the technique of choice regarding its reliability and effectiveness particularly seen in pelvic resection type I and IV. Nevertheless, mechanical failure still common among variation techniques using this system and very little studies to dates compare effectiveness among those. ${ }^{5}$

None has studied the use of PSRS for pelvic reconstruction following pelvic resection type II or III. Kamal et al. Recommends arthrodesis or arthroplasty for pelvic reconstruction in resection type II. The principle of arthrodesis after pelvis resection is to achieve solid union between proximal femur and the remaining pelvis which can achieved by using any kind of fixation devices such as plate, screw, or wire. However, the disadvantage of arthrodesis includes loss of the hip joint function which is disabling and reduced functional outcome. Fusion rates of femoro-pelvic arthrodesis are less than 50\%, in most patient, a stable and painless pseudo-arthrosis develops, but with a comparable functional result to that of the alternative reconstruction options. Arthroplasty reconstructions are recommended when remaining ilium and pubis are sufficient for fixation of pelvis allograft or autograft composite with a unipolar or total hip prosthesis. ${ }^{1}$

Zou et al. recommend the use of PSRS for reconstructive surgery after I or I + IV pelvic resection and for other indications such as isolated metastasis tumor, reserving the top of the acetabulum of the pubis and sciatic after tumor excision. Their study showed that PSRS is a safe method that has rapid rehabilitation, shorter hospital stays, lower complication rates and favorable functional results with most patients achieving independent ambulation and excellent cosmetics. But this method is technology contraindicated to the isolated areas II or III of pelvic primary tumors because often it cannot guarantee the stability of the pedicle screw-rod system or complete cutting edge after resection of the tumor. ${ }^{13}$

We presented the technique of hinged arthrodesis with PSRS following pelvic resection type 1/ II/III. Although very little evidence and an article of zou et al. Not recommend the use of PSRS following pelvic resection type II/III, we aim to achieve its characteristics such as easy manipulation, few complication and stable fixation. We elaborate the technique utilizing 4 monoaxial pedicle screws to provide stable fixation within the pelvicarch and 2 polyaxial pedicle screws to allow movement at the hip joint. We didn't use biological graft in addition to the system to reduced infection and non-non union rate. The pre-bendrods are designed to transmit loads both in sitting and standing. The pedicle screw usage is relatively simple rather than screws in ORIF composite regarding less stress concentration due to multi-point fixation of screws as seen in ORIF composite.

Post operatively, as expected patient could regain 0-45 degree of hip flexion which are not seen in traditional hip arthrodesis. Patient Satisfactory increased because we still preserved her limb srather than ablating the remaining functional unit of lowerlimb. Patient also happy and not experiencing psychological trauma due to the poor aesthetic outcome after ablation. Complication did occur regarding 
the sciatic nerve palsy which has been informed preoperatively. It is extremely difficult to preserve the nerve intraoperatively since the mass has been infiltrated through the sciatic notch.

Infection is frequent following pelvic reconstruction particularly if using grafts or prosthesis. Several conditions can contribute to the risk of infection after pelvic reconstruction such as immuno compromise (regarding the patient nutrition or preoperative neoadjuvant chemotherapy), prolonged surgical time, proximity to visceral organs such as rectum and genito-urinary tract, large dead space after tumor removal and large amount of implant which is considered as foreign materials implanted in operated area. Some authors recommend the use of local high concentrations of antibiotic released from antibiotic-impregnated cement is the most important factor in the infection prevention. In our case, we used simpler and lesser implant and applied local antibiotic bone cement combined with systemic antibiotic administration. Our patient did not experience infection post operatively. The hinged PSRS we used in this patient also encouraged her for early mobilization and thus decreased hospital length of stay, increased independency and improved her quality of life as observed in MSTS score.

Our study has some limitation such as the need of more case using this system and the need of longer time of follow up to observe any mechanical failure of the system and the tumor related complication such as recurrence or metastases.

\section{Conclusion}

Treatment of pelvic tumor by limb salvage procedure is a developing area of orthopedic oncology surgeon. Variation of surgical options has been proposed to overcome the large defect and reconstruct the stable pelvic arch for its major function as weight bearing surface. The use of a pedicle screw-rod system after resection of malignant pelvic tumor is an acceptable method of pelvic reconstruction because of its lower rate of complications and satisfactory functional outcome. Technically, it is relatively simple to be applied, no special instrumentation needed, and easy to be manipulated. We provided an evidence of a case treated with pelvic resection type I/II/III with good outcome seen in 6 months and 1 year follow up. We suggested that this method is a good option to consider when treating the patient with similar type of pelvic resection.

\section{Acknowledgments}

None.

\section{Funding}

None.

\section{Conflict of interest}

There is no conflict of interest.

\section{References}

1. Kamal AF, Prabowo Y, Wahyudi M. Outcomes of pelvic resection from malignant pelvic tumors. A case series. IJSO. 2019(16):34-39.

2. Puchner SE, Funovics PT, Böhler C, et al. Oncological and surgical outcome after treatment of pelvic sarcomas. PLos One. 2017;15:12(2):e0172203.

3. Guo W, Sun X, Ji T, et al. Outcome of surgical treatment of pelvic osteosarcoma. J Surg Oncol. 2012;106(4):406-410.

4. Schwameis E, Dominkus M, Krepler P, et al. Reconstruction of the pelvis after tumor resection in children and adolescents. Clin Orthop Rel Res. 2002;402:220-35.

5. Lin P, Shao Y, Lu H, et al. Pelvic reconstruction with different rod-screw systems following Enneking type I/I + IV resection: a clinical study. Oncotarget. 2017;8(24):38978-38989.

6. Guo Z, Li J, Pei G-X, Li X-D, et al. Pelvic reconstruction with a combined hemipelvic prostheses after resection of primary malignant tumor. Surg Oncol. 2010;19(2):95-105.

7. Mayerson JL, Wooldridge AN, Scharschmidt TJ. Pelvic resection: Current concepts. J Am Acad Orthop Surg. 2014;22(4):214-222.

8. Apffelstaedt JP, Driscoll DL, Spellman JE, et al. Complications and outcome of external hemipelvectomy in the management of pelvic tumors. Ann Surg Oncol. 1996;3(3):304-309.

9. Aljassir F, Beadel GP, Turcotte RE. Outcome after pelvic sarcoma resection reconstructed with saddle prosthesis. Clin Orthop Relat Res. 2005;438:36-41.

10. Angelini A, Calabro T, Pala E, et al. Resection and reconstruction of pelvic bone tumors. Orthopedics. 2015;38(2):87-93.

11. Enneking WF, Dunham WK. Resection and reconstruction for primary neoplasms involving the in nominate bone. J Bone Joint Surg Am. 1978;60(6):731-746.

12. A AY, Ek ET, Choong PF. Research: is resection of tumours involving pelvic ring justifed? A review of 49 consecutive cases. Int Semin Surg Oncol. 2005;2(1):9.

13. Zhou YJ, Yunus A, Tian Z, et al. The pedicle screw-rod system is an acceptable method of reconstructive surgery after resection of sacroiliac joint tumours. Contemp Oncol. 2016;20(1):73-79. 